\title{
The Sailboat Exercise as a Method for User Understanding and Requirements Gathering
}

\author{
Paula Alexandra Silva \\ Department of Informatics Engineering | Centre for Informatics and Systems \\ (CISUC), University of Coimbra, Coimbra, Portugal paulasilva@dei.uc.pt
}

\begin{abstract}
To design digital products and services that truly empower end-users requires that design and development teams involve end-users early and throughout the design process. However, regardless of the wealth of methods available to Human-Computer Interaction designers, to identify tools that are both intuitive to use and allow for the active engagement of end-users, namely though co-design activities, is hardly ever easy. To identify a simple and straightforward method can be challenging especially when the end -user group are older adults. This paper proposes an adaptation of an exercise, traditionally used in agile retrospectives - the sailboat exercise - here modified and tailored to be used as a co-design generative tool for user understanding and requirements gathering. In short, the method leverages the analogy of a sailboat, and its surrounding factors, and combines it with a set of prompt questions, to create a shared understanding between the end-users and the members of the design team and to support identification of users' goals, desires, challenges and frustrations.
\end{abstract}

\section{Keywords}

User research $\cdot$ Co-design $\cdot$ Participatory design method

How to cite this book chapter:

Silva, P.A. 2020. The Sailboat Exercise as a Method for User Understanding and

Requirements Gathering. In: Loizides, F., Winckler, M., Chatterjee, U., Abdelnour-Nocera, J. and Parmaxi, A. (eds.) Human Computer Interaction and Emerging Technologies: Adjunct Proceedings from the INTERACT 2019 Workshops. Pp. 31-42. Cardiff: Cardiff University Press. DOI: https://doi.org/10.18573/ book3.d. License: CC-BY 4.0. 


\section{Introduction}

Including end-users in the design process allows for effective requirements gathering and increases both user satisfaction and the level of acceptance of the final design [1]. Therefore, it is key to practice a co-design approach, if aiming at novel [2], differentiated and inclusive solutions [3] and at designing digital products and services that do not fail to be adopted when market ready $[2,4]$.

Users are experts of their own experience [3] and as such, potential end-users should actively contribute throughout the design process as domain experts, working in cooperation, as equal partners, with the design team [5]. This generally involves engaging in a number of collaborative activities, the so-called 'generative tools' [5] or 'tools for conversation' [6], that allow users and stakeholders to dialogue and contribute their views, insights, and feedback.

User involvement is important throughout the design process (Fig. 1), at all stages, but it is vital in earlier phases. The specification of the context of use and of the user requirements are the first phases of human-centred design [7], thus impacting all sub sequent phases of the design of digital products and services.

From interviews, questionnaires, and focus groups to diary studies, photovoice, and workshops, there are many methods that can be used with the purpose of developing an understanding of the user and the context of use and for gathering and specifying user requirements (for methods descriptions refer to [8-11]). However, when designing with older adults, several adaptations may be required, ranging from simple communication and language adjustments to the need for meeting older adults at their own home [11-13]. Furthermore, especially when referring to the design of interactive systems, negative experiences, attitudes, assumptions, and preconceptions of both older adults and design teams regarding older adults and technology may get in the way, adding to the challenge of doing user research with this user group [11]. Design teams need to be creative and resourceful, carefully choosing and/or developing methods and tools that are both straightforward to use, requiring minimal instructions, and that enable 'conversations', around which user needs are made explicit from which key design insight can be gathered [6].

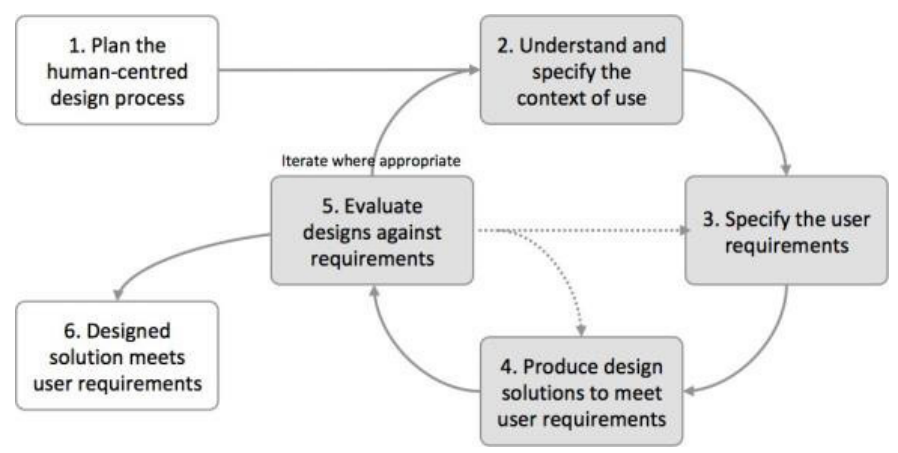

Fig. 1: User-centered design process, adapted from ISO 9241-210:2010 [7]. 
This paper introduces a modified version of the sailboat exercise, traditionally used in agile retrospectives [14], as a method for user understanding and requirements gathering that supports the process of making user goals, desires, challenges and frustrations explicit, while still keeping this process easy to understand and use. The following sections provide a concise background on the origins of the method and present its modified version. The paper then provides instructions for facilitation and elaborates on the specific value of the exercise.

\section{The Sailboat Exercise}

\subsection{Original Version of the Sailboat Exercise}

The sailboat exercise is credited to Ben Linders [15] and is commonly used by agile teams applying scrum processes. In the scope of agile retrospectives, "a retrospective is a regular meeting during which the team reflects on how team members work together and considers ways to improve that process, based on lessons from recently completed work." [14]. These meetings take place at the end of each cycle or iteration and are meant to create a platform for discussion and reflection on the team's successes and failures. In this process, the team identifies what went well, so that such aspects are repeated, and what failed, so that the team learns from what went wrong and sets out improvement strategies. All team members are invited to participate and contribute feedback. At the end of a retrospective, the team creates an action plan for addressing improvements, listing the next steps and assigning e ach step an owner, date, and priority level; subsequently the action plan is made available to all [14].

When using the sailboat exercise, a picture of a sailboat (Fig. 2) is used to facilitate the meeting discussion. Although a retrospective does not necessarily need to use the sailboat exercise, by using the analogy of the sailboat, it is easier for a team to relate to what is working well, propelling them forward, and what is holding them back.

\subsection{Modified version of the Sailboat Exercise}

Retrospectives have been used by product design teams (e.g. when launching a new product), user experience design teams (e.g. when introducing a new method), and leadership teams (e.g. when implementing a new company initiative or strategic plan) [14]. Here the sailboat exercise is proposed as a tool to engage end-users, and other stakeholders involved in the design process, in a shared activity that aims at supporting the elicitation of user requirements. This method has been used as part of codesign training workshops for nondesigners [16]. The next section describes the structure of the proposed modified version of the method. 


\title{
The sailboat exercise
}

\author{
The boat / trade winds.
}

\section{The anchor}

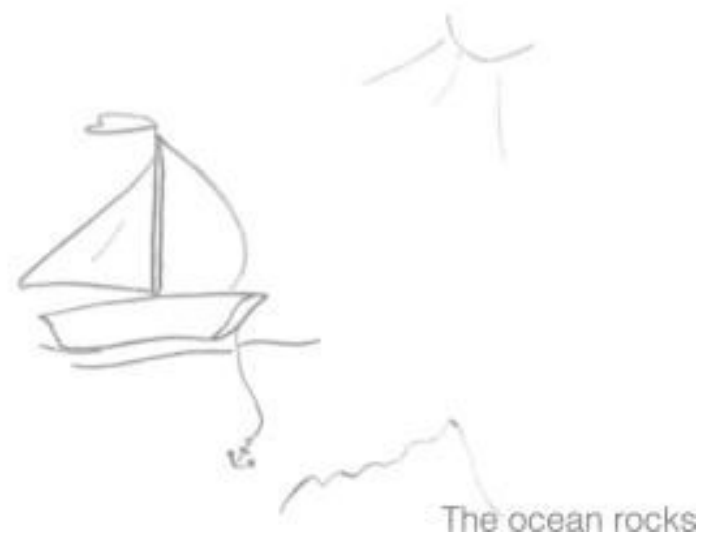

Fig. 2: Sailboat exercise support sheet.

Structure of the Method. While the original version of the sailboat exercise is intended for reflecting on a team's performance, in this article, the sailboat exercise is proposed as a method for understanding end-users and elicitation of their goals, desires, challenges, and frustrations. Additionally, while in its original version, the sailboat exercise is mainly directed at identifying plusses and minuses within the team's processes, this article proposes that all four sailboat surrounding factors - Boat/Trade Winds; Sun/Land; Anchor; and Ocean Rocks-are used to truly harness the full potential of the sailboat analogy. Specifically, it is proposed that:

- The Boat/Trade Winds factor is linked to user Goals, i.e. aspects of an experience that are currently available, working well, and allow the user to achieve her/his goals, e.g. the convenient bus stop outside her/his apartment door;

- The Sun/Land factor is linked to user Desires, i.e. anything that allows for a perfect effortless experience to become a reality, e.g. a 24/7 chauffeur;

- The Anchor factor is linked to user Frustrations, i.e. any personal circumstances that are holding a person back or creating difficulties, e.g. the persistent pain of a past hip fraction;

- The Ocean Rocks factor is linked to user Challenges, i.e. any external factors that are creating obstacles, e.g. a bus that is not prepared for wheelchair users to hop on, or the absence of bike lanes in the area.

In addition, it is proposed that the sailboat analogy is complemented by four sets of prompt questions, each associated to one of the sailboat 


\section{Sailboat exercise: prompt questions}

\begin{tabular}{|ll|}
\hline The boat / trade winds & The sun / land \\
- What's working well at the & - What would the truly perfect \\
moment? & experience look like? \\
- Anything that helps achieving & - What are you striving towards? \\
your goal. & - Things, qualities, features that \\
- Things, skills, qualities currently & would create/contribute to an \\
available. & ideal, desirable situation. \\
& \\
& \\
The anchor & The rocks \\
- What's holding you back? & - What obstacles hold outside \\
- Aspects and/or situations, & which are preventing you from \\
which are making things & achieving our goal? \\
difficult, slowing you down. & - Problems you find along the \\
& way. \\
\hline
\end{tabular}

Fig. 3: Sailboat exercise prompt questions.

surrounding factors (Fig. 3). The prompt questions are meant to assist in the elicitation of beneficial and/or detrimental experiential aspects, by triggering participant to make them explicit. The prompt questions further intend to facilitate communication and reasoning while adding focus and direction to the exercise. Other methods have also used prompt questions to facilitate design-related processes [17].

Table 1, column 'Procedures', describes the steps involved in conducting a session with the sailboat exercise. Once all steps are completed, each group should have produced a list of items, organized under four categories, identifying the top three items to prioritize.

How the Method Fits in the Design Process. At the end of a sailboat exercise session, the design team collects the outcomes from all groups and reorganizes the results, by grouping similar or duplicate ideas together creating an aggregated list. Once this list exists, it is possible to create a list of user interface specifications, by converting user produced materials into a formal list of user interface design requirements and specifications. It is important not to lose track of the items identified, and negotiated by the team, as most relevant, as these are likely to indicate the features that should be core in the product or service to be developed. Thus the outcomes of the Sailboat exercise make an important contribution to informing the design, in particular phases 2 and 3 of the design process (Fig. 1). From the aggregated list of requirements and specifications, it is possible to proceed to the ideation phase in which design solutions are prototyped and produced. 
Table 1: The sailboat exercise list of procedures and preparation guidelines.

Preparation
Total number of participants: $16-20$

Number of participants per group: $4-8$

Time: 90-180 minutes; length will vary with level of complexity of the design brief and participants' skills.

\section{Room and materials:}

- Reserve a quiet and comfortable room that allows for discussion, writing, and privacy.

- One A4 print out of the sailboat worksheet per participant.

- One A3 print out of the sailboat worksheet sheet per group.

- One set of small sized post-its (ideally in diverse colors, each assigned to a different category: goals, desires, challenges, and frustrations) per participant, for each to write down ideas.

- Pens and pencils for each participant.

- Four sets of five sticky dots/stars per participant, for voting.

- Give each group a name, number or letter.

- Printout of the group name.

- Printout of the design brief/context.

- Printout of the prompt questions.

\section{Optional}

- Printout of 'motivate to action' statements.

- Printout of rules for ideation per group.

- Printout of rules for discussion.

\section{Before the session starts:}

- Develop a clear description of the design challenge, stating the design domain and challenge the participants will work on.

- Remember to prepare and collect informed consent from all participants.

- You may wish to bring a soundtrack (there are plenty available online, some even

- with a timer) to play in the background as participants go through the steps of the exercise.

\section{Procedures}

Part 1 - Starting off (20 minutes):

- Present the design brief.

- Explain how the method works, clarifying what the trade wind, anchor, rocks, and sun mean and the purpose of the prompt questions.

- Name (or ask each group to) a rapporteur.

- Ask each participant to write down her/his name on her/his A4 sailboat worksheet.

- Ask the rapporteur to write group name, number or letter on their A3 sailboat page.

\section{Part 2 - Individual sailboat (10 minutes):}

- Have each participant record her/his goals, desires, challenges, and frustrations, by writing them down directly on the A4 sailboat worksheet or by using post-its.

- Have each participant present her/his ideas to the team.

\section{Part 3 - Group sailboat (30 minutes):}

- As each participant presents her/his ideas, the rapporteur records them on the group A3 the sailboat worksheet (alternatively participants can simple move in the post-its they previously used).

- Once contributions are gathered and grouped by category, have the team discuss the results and agree on which ideas are most relevant.

- In case there is a large number of ideas and the group does not naturally come to consensus, invite participants to use the five sticky dots and vote for the three ideas they would most like to see prioritized.

\section{Part 4 - Debriefing (30 minutes):}

- Allow each team to present the results and speak about their list to all participants. 


\begin{tabular}{ll}
\hline Preparation & Procedures \\
\hline Total number of participants: 16-20 & Part $\mathbf{1}$ - Starting off (20 minutes): \\
- In room: & - Keep it brief and genuine, but make \\
- Form (or ask participants to) the & sure to acknowledge participants' \\
teams, assigning profiles evenly, if & contributions and express thanks. \\
stakeholders are in the room. & Important to keep in mind during \\
- Observe the participants and check if & session: \\
anyone needs help writing, reading, & - Remind participants there are no right \\
hearing, etc. & or wrong answers. \\
& - When debriefing, distribute available \\
& time evenly across participants \\
& and teams. \\
& - As a facilitator, remember to keep a \\
& low profile; the same applies to any \\
& helpers. \\
& - Capture all notes and make photos of \\
& the session and the materials created. \\
\hline
\end{tabular}

In this process, the Boat/Trade Winds or user Goals are features the design team will want to keep; the Sun/Land or user Desires consist of opportunities for improvement; the Anchor or user Frustrations are likely to correspond to aspects the design needs to accommodate for; and the Ocean Rocks or user Challenges represent factors that need to be resolved and could give the product or service a strategic advantage.

Planning for and Facilitating a Session with the Sailboat Exercise. Similar to other methods, when planning for a session with the sailboat exercise, preparing and clarifying both the session's activities and the design brief is essential.

Essentially the design brief should: i) clarify the design domain, i.e. the broad area in which the group is going to work, and ii) concisely describe the design problem or challenge that is going to be addressed. The design domain and design challenge should be written in a clear, concise statement ${ }^{1}$. During the session this statement should be visible and it is recommended that the facilitator complements the written statement with examples and further details. In addition to the design brief, the facilitator should arrange for a suitable venue in which to conduct the exercise and for various supplies (for a full list of requirements please refer to the column 'Preparation' in Table 1).

\section{The Value of the Sailboat Exercise}

The design of digital products and services which meet user needs and are accepted when market-ready, demands careful consideration of end-users' needs and their active involvement in the design process $[1,2]$. In this

${ }^{1}$ An example of a possible statement could be 'Getting places, when I can no longer drive'. 
context, it is important to create opportunities for collaboration, communication, and exchange, which can spur design knowledge. The sailboat exercise allows for the active engagement of end -users and can be easily adapted to different audiences and design problems. In addition to being adaptable across different contexts, the sailboat exercise has several other benefits, which are discussed in the following three sections.

\subsection{A tool participants relate to immediately}

One of the advantages of the sailboat exercise stems from its simple analogy. To establish a parallel between the impact of goals, desires, frustrations and challenges on user experience and the impact of the four factors-Boat/Trade Winds, Sun/Land, Anchor, Ocean Rocks-on a sailboat is simple and straightforward. This makes it easy to create a shared understanding about the activity and to rapidly engage in a conversation where users are able to relate to goals, desires, frustrations, and challenges, without requiring long and complex explanations.

\subsection{A tool that allows for different stakeholders to be involved}

All types of stakeholders, from older adults to formal or informal caregivers can be invited to participate in a sailboat exercise session. This means that the design team can simultaneously collect needs from the different stakeholders, while still having each participant making her/his own individual needs explicit (Part 2 of the method). In Part 3, the full potential of the method is harnessed, when the participants, as a team, negotiate and make decisions on what requirements should be prioritized to create one single list. This allows the design team to gain an understanding of the diverse requirements of (related) users and their complete context of use, providing a more accurate representation of the context. To involve the different stakeholders in eliciting and negotiating requirements has been identified as key in projects that aim to be innovative [18].

\subsection{A tool that allows for easy follow-ups}

A Human-Computer Interaction (HCI) design process is iterative. This means that an HCI design team may need to revisit design requirements throughout the design process and that multiple meetings to review requirements may be necessary. Repeating or carrying out multiple data gathering sessions also helps to ensure accurate interpretation. Users may not be used to articulating their needs and may use or assign meaning to terms differently from how those terms are used in design fields; therefore, it is important to review each session and check interpretations with users/stakeholders to ensure clarity. 


\section{Conclusions}

To design appropriate digital products and services it is vital to understand end -users and to grasp what they want to do with the products/services as well as the environments in which the products /services will be used. This paper introduced the sailboat exercise as a method for improving understanding of end -users and the contexts in which they are immersed. The exercise can be facilitated by designers with end -users/stakeholders at the early stages of $\mathrm{HCI}$ design processes. The proposed method demonstrates potential for aiding with the important task of user requirements gathering-it is flexible, requires little to moderate preparation and is accessible in form and content for a variety of people. As reported in [16], the method has been successfully used to assist requirements gathering of Active and Assisted Living systems, but in the future it would be interesting to experiment how it performs in other domains.

\section{Acknowledgments}

This research was developed with the support of the Research Program "CeNTERCommunity-led Territorial Innovation” (CENTRO-01-0145-FEDER-000002), funded by Programa Operacional Regional do Centro (CENTRO 2020), PT2020. Many thanks also to Leah Burns, Aalto University, for the suggestions on the manuscript.

\section{References}

1. Kujala, S.: User involvement: A review of the benefits and challenges. Behaviour \& Information Technology. 22, 1-16 (2003). doi:10.1080/01449290301782

2. Trischler, J., Pervan, S.J., Kelly, S.J., Scott, D.R.: The Value of Codesign: The Effect of Customer Involvement in Service Design Teams. Journal of Service Research. 21, 75-100 (2018). doi:10.1177/1094670517714060

3. Co-Create Project: Co-Create Essentials. (2019)

4. Wherton, J., Sugarhood, P., Procter, R., Hinder, S., Greenhalgh, T.: Coproduction in practice: how people with assisted living needs can help design and evolve technologies and services. Implementation Science. 10, 75 (2015). doi:10.1186/s13012-015-0271-8

5. Sanders, E.B.-N., Stappers, P.J.: Co-creation and the new landscapes of design. CoDesign. 4, 5-18 (2008). doi:10.1080/15710880701875068

6. Sanders, E.B.-N.: Generative Tools for Co-designing. In: Scrivener, S.A.R., Ball, L.J., and Woodcock, A. (eds.) Collaborative Design. pp. 3-12. Springer London, London (2000) 
7. ISO 9241-210:2010(en), Ergonomics of human-system interactionPart 210: Human-centred design for interactive systems, https://www.iso .org/obp/ui/\#iso:std:52075:en

8. Preece, J., Sharp, H., Rogers, Y.: Interaction Design: Beyond HumanComputer Interaction. Wiley, Chichester (2015)

9. Farrell, S.: UX Research Cheat Sheet, https://www.nngroup.com/articles /uxresearch-cheat-sheet/

10. Rohrer, C.: When to Use Which User-Experience Research Methods, https://www.nngroup.com/articles/which-ux-research-methods/

11. Eisma, R., Dickinson, A., Goodman, J., Syme, A., Tiwari, L., Newell, A.F.: Early user involvement in the development of information technologyrelated products for older people. Universal Access in the Information Society. 3, 131-140 (2004). doi:10.1007/s10209-004-0092-z

12. Silva, P.A., Nunes, F.: $3 \times 7$ Usability Testing Guidelines for Older Adults. In: MexIHC 2010 (2010)

13. Dickinson, A., Arnott, J., Prior, S.: Methods for human-computer interaction research with older people. Behaviour \& Information Technology. 26, 343-352 (2007). doi:10.1080/01449290601176948

14. Krause, R.: UX Retrospectives 101, https://www.nngroup.com/articles /uxretrospectives/

15. Gonçalves, L.: Run the Sailboat Agile Exercise or Sailboat Retrospective, https://medium.com/@lgoncalves1979/sailboat-exercise-sailboat -retrospective-4893ca6b5fd3, (2017)

16. Silva, P.A., Daniel, A.D.: Training Non-Designers in Co-Design Methods through an Active Assisted Living Interactive Workshop. In: Human-Computer Interaction - INTERACT 2019. Springer International Publishing. (2019)

17. Silva, P.A.: BadIdeas 3.0: A Method for Creativity and Innovation in Design. In: Proceedings of the 1st DESIRE Network Conference on Creativity and Innovation in Design. pp. 154-162. Desire Network, Aarhus, Denmark (2010)

18. Fitzpatrick, G., Malmborg, L.: Quadruple Helix Model Organisation and Tensions in Participatory Design Teams. In: Proceedings of the 10th Nordic Conference on Human-Computer Interaction. pp. 376-384. ACM, New York, NY, USA (2018) 